\title{
In-plane resistivity in graphite intercalation compounds obtained from conduction-electron-spin-resonance measurements
}

\author{
L. Walmsley \\ Departamento de Física, Instituto de Geociencias e Ciencias Exatas, Universidade Estadual Paulista, \\ C.P. 178, CEP 13500, Rio Claro, São Paulo, Brazil \\ (Received 6 December 1991; revised manuscript received 23 March 1992)
}

\begin{abstract}
We developed a procedure to take advantage of the magnetic-field-modulation-frequency effect on the line shape of conduction-electron-spin resonance of graphite intercalation compounds (GIC's) to extract the absolute value of the in-plane resistivity. We calculated the power absorbed in each slice of the sample normal to the wave penetration, multiplied by a factor to account for the magnetic-field-modulationfrequency effect. Room-temperature spectra of stage- $1 \mathrm{AlCl}_{3}$-intercalated GIC in both $\mathbf{H}_{0} \perp c$ and $\mathbf{H}_{0} \| c$ configurations were fitted to the theoretical line shapes and the value of in-plane resistivity (and also the value of $c$-axis resistivity) obtained from the fitting parameters are in reasonable agreement with those from the literature.
\end{abstract}

\section{INTRODUCTION}

Graphite intercalation compounds (GIC's) have been extensively studied by means of conduction-electron-spin resonance (CESR). The room-temperature spectra are in general very intense, showing a Dysonian line shape and an isotropic $g$ value. ${ }^{1}$

Due to the large resistivity anisotropy of GIC's ( $\rho_{c} / \rho_{a} \sim 10^{5}-10^{6}$ in acceptor compounds) care must be taken in choosing the sample dimensions in order to correctly analyze the electrical transport data obtained from the Dysonian line shape.

In Fig. 1 we have a typical GIC sample of height $h$, width $l$ and thickness $d$. We see that the microwave magnetic field $\left(\mathbf{H}_{\mu}\right)$ penetrates by the $(h, d)$ faces. Since $\mathbf{E}_{\mu}$ is parallel to the $c$ axis, the skin depth is governed by the $c$ axis resistivity $\left(\delta_{c}^{\mu}\right)$. We will use $I_{c}$ to denote the intensity of the resonance observed in this configuration. Microwaves can also come from $(l, h)$ faces, in which case, the skin depth is governed by the in-plane resistivity $\left(\delta_{a}^{\mu}\right)$ and we will use $I_{a}$ to denote the observed resonance intensity. We neglect the intensity of the resonance corresponding to microwave penetrating from the small $(d, l)$ surfaces. Sample thickness and resistivity anisotropy will determine the most important contribution to the resonance intensity. We can therefore use $I_{a} / I_{c}=\left(l \delta_{a}^{\mu}\right) /\left(d \delta_{c}^{\mu}\right) \gg 1\left(I_{a} / I_{c}<<1\right)$ as a criterion for associating the observed resonance with $I_{a}$ (thin sample) $\left[I_{c}\right.$ (thick sample)]. ${ }^{2}$

If the resonance is associated with $I_{a}$, we can analyze the line shape in terms of Dyson's one-dimensional theory, ${ }^{3}$ taking Dyson's parameters $\lambda=d / \delta_{a}^{\mu}$ and $R=\delta_{a}^{\mu} /\left(2 D_{c} T_{2}\right)^{1 / 2}, D_{c}$ being the diffusion constant along the $c$ axis and $T_{2}$ the spin relaxation time. In this case since $d \gg \delta_{a}^{\mu}$ the application of Dyson's theory allows only the determination of $R$ (and $D_{c}$ ) from the Dysonian line shape. ${ }^{1}$

If the resonance is associated with $I_{c}$, we can use Dyson's one-dimensional theory, taking Dyson's parame- ters $\lambda=1 / \delta_{c}^{\mu}$ and $R=\delta_{c}^{\mu} /\left(2 D_{a} T_{2}\right)^{1 / 2}, D_{a}$ being the inplane diffusion constant. Now we can have $l \sim \delta_{c}^{\mu}$ and the application of Dyson's theory to the line shape yields either $\lambda$ (if $\lambda \leq 2.5$ ), or $\lambda$ and $R$ (if $2.5 \leq \lambda \leq 8$ for $R \geq 1$ or $2.5 \leq \lambda \leq 30$ for $R \leq 0.5$ ). A more complete discussion of the effects of geometry and configuration on the CESR line shape of GIC's can be found in Ref. 2 .

In studying the CESR line shapes of $\mathrm{AsF}_{5}$-intercalated GIC's, Saint Jean et al. ${ }^{4}$ reported the line shape dependence on the magnetic-field modulation frequency in the configuration in which the static magnetic field is parallel to the $c$ axis of GIC's $\left(\mathbf{H}_{0} \| c\right)$. The line shape in the configuration $\left(\mathbf{H}_{0} \| c\right)$ differs from that in the configuration $\left(\mathbf{H}_{0} \perp c\right)$ for high values of the magnetic-field modulation frequency. In contrast, for low values the line shapes for both configurations coincide.

In Ref. 2 we qualitatively explained this effect that can be observed only in thick samples of GIC's with highresistivity anisotropy. It can be understood if one looks at the configuration of Fig. 1 (for thick samples) to ob-

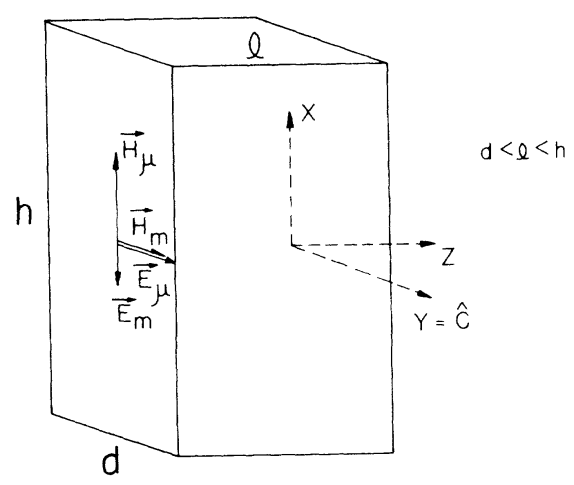

FIG. 1. Configuration of microwave electric $\left(\mathbf{E}_{\mu}\right)$ and magnetic $\left(\mathbf{H}_{\mu}\right)$ fields and modulation electric $\left(\mathbf{E}_{m}\right)$ and magnetic $\left(\mathbf{H}_{m}\right)$ fields. We assume that the penetration occurs only in the direction parallel to the width of the sample ( $z$ direction). 
serve microwave penetrating with skin depth $\delta_{c}^{\mu}$. The slow oscillating $\left(v_{m} \leq 100 \mathrm{kHz}\right)$ modulation magnetic field $\left(\mathbf{H}_{m}\right)$ will always be parallel to the static magnetic field $\mathbf{H}_{0}$. Consequently, the penetration of the modulation field will be determined by $\delta_{c}^{m}$ for $\mathbf{H}_{0} \perp c$ and by $\delta_{a}^{m}$ for $\mathbf{H}_{0} \| c$. For $\mathbf{H}_{0} \perp c$, the skin depth for the magnetic-field modulation frequency of $100 \mathrm{kHz}$ will always be larger than usual sample dimensions $\left[\delta_{c}^{m}(100 \mathrm{kHz})>30 \mathrm{~mm}\right]$ and so the sample will be homogeneously modulated and no magnetic-field modulation frequency effect will be observed. But for $\mathbf{H}_{0} \| c$ and frequencies in the range $10-100 \mathrm{kHz}$, we have $\delta_{a}^{m} \lesssim \delta_{c}^{\mu} \sim l$ and the detected signal arises from an inhomogeneously modulated sample. The higher the modulation frequency, the stronger is the effect. As it is expected, the line intensity is also affected, decreasing with increasing modulation frequency. In this work, we developed a procedure to take into account quantitatively the magnetic-field-modulation-frequency effect on the CESR line shape so as to obtain the in-plane resistivity $\rho_{a}$.

\section{DESCRIPTION OF THE METHOD}

In order to obtain the CESR line shape of a thick GIC sample, we use Kaplan's theory ${ }^{5}$ applied for a symmetrical penetration of microwave field. This theory gives the same results as Dyson's and is easier to handle. Both theories calculate the power absorbed by the sample by taking the real part of the complex Poynting vector taken just outside the surface of the sample. For obtaining the frequency-modulated power we need the power absorbed in each slice of the sample normal to the microwave (and modulation field) penetration direction. This power is multiplied by a factor to take the magnetic-field modulation frequency into account, having each slice of the sample contributing to the line shape with a different weight. Thus the line shape must be obtained from a volume and not from a surface calculation. This can be accomplished by using the Poynting theorem:

$$
\int_{S} \mathbf{S} \cdot \mathbf{n} d a=-\frac{\partial}{\partial t} \int_{v} u d v-\int_{v} \mathbf{J} \cdot \mathbf{E} d v,
$$

where $\mathbf{S}=(c / 4 \pi)(\mathbf{E} \times \mathbf{H})$ is the Poynting vector, $u=(1 / 8 \pi)(\mathbf{E} \cdot \mathbf{D}+\mathbf{B} \cdot \mathbf{H})$ is the density of electromagnetic energy, and $\mathbf{J} \cdot \mathbf{E}$ is the Joule heat.

In Fig. 1 we show the configuration of the microwave fields $E_{y}$ and $H_{x}$ in a typical GIC sample. We assume the spatial variation of the fields in the $z$ direction only. For our GIC sample we have $H_{y}=E_{x}=0$ but we can write in a general form:

$$
u_{M}(z)=\frac{1}{8 \pi}\left[B_{x}(z) H_{x}(z)+B_{y}(z) H_{y}(z)\right],
$$

where $B_{x}(z)=H_{x}(z)+4 \pi M_{x}(z)$.

For obtaining $H_{x}(z), M_{x}(z), H_{y}(z)$, and $M_{y}(z)$ we solve the same equations that Kaplan did for the transverse components of magnetization, together with Maxwell equations neglecting the displacement current:

$$
\begin{aligned}
& \frac{\partial \mathbf{M}}{\partial t}=\gamma \mathbf{M} \times \mathbf{H}-\frac{\mathbf{M}}{T_{2}}+D \nabla^{2} \mathbf{M}, \\
& \nabla \times \mathbf{E}=-\frac{1}{c}\left(\frac{\partial \mathbf{H}}{\partial t}+\frac{\partial \mathbf{M}}{\partial t}\right), \\
& (\nabla \times H)_{i}=\frac{4 \pi}{c} \sum_{j} \sigma_{i j} E_{j},
\end{aligned}
$$

where $\gamma$ is the gyromagnetic ratio, $T_{2}$ is the spin relaxation time, $D=D_{a}$ is the in-plane diffusion constant, and $\sigma_{i j}$ is the conductivity tensor.

Considering the $x$ and $y$ components of $\mathbf{M}, \mathbf{H}$, and $\mathbf{E}$ varying in a symmetric form as $e^{i \omega t} \cosh (k z)$ and defining

$$
\begin{gathered}
M=M_{x}-i M_{y}, \quad \mathscr{H}=H_{x}-i H_{y}, \quad R^{2}=\delta^{2} /\left(2 D T_{2}\right), \\
K^{2}=k^{2} \delta^{2} /\left(2 R^{2}\right), \quad \alpha=T_{2}\left(\omega-\omega_{0}\right), \quad \beta=\chi_{0} \omega_{0} T_{2},
\end{gathered}
$$

where $\delta=\delta_{c}^{\mu}$ is the microwave skin depth governed by $c$ axis resistivity and $\chi_{0}$ is Pauli susceptibility.

Equations (1) are solved to give the values of $K^{2}$ :

$$
K_{1}^{2}=\left(c_{1} / 2\right)\left[1+\left(1-4 c_{2} / c_{1}^{2}\right)^{1 / 2}\right]
$$

and

$$
K_{2}^{2}=\left(c_{1} / 2\right)\left[1-\left(1-4 c_{2} / c_{1}^{2}\right)^{1 / 2}\right],
$$

where

$$
c_{1}=1+i\left(\alpha+1 / R^{2}\right)
$$

and

$$
c_{2}=\left(1 / R^{2}\right)[4 \pi \beta+i(1+i \alpha)] .
$$

For symmetric penetration we write $\mathscr{H}(z)=\mathscr{H}_{1}+\mathscr{H}_{2}$ $=A_{1} \cosh \left(k_{1} z\right)+A_{2} \cosh \left(k_{2} z\right)$. Since for this field the magnetization is linear with the field, we write

$$
M=M_{1}+M_{2}
$$

where

$$
M_{n}=v_{n} \mathscr{H}_{n}
$$

and

$$
v_{n}=-i\left(K_{n}^{2}-i / R^{2}\right) /\left(4 \pi / R^{2}\right) .
$$

For the geometry described in Fig. 1 we apply boundary conditions

$$
\mathscr{H}(z=-l / 2)=\mathscr{H}_{10}
$$

and

$$
\frac{\partial M}{\partial z}(z=-l / 2)=0,
$$

where $\mathscr{H}_{10}$ is the microwave magnetic field at the surface of the sample. We obtain

$$
A_{1}=\frac{\mathscr{H}_{10}}{\left\{\cosh \left(k_{1} z\right)\left[1-\left(v_{1} k_{1} / v_{2} k_{2}\right) \tanh \left(k_{1} l / 2\right) \operatorname{coth}\left(k_{2} l / 2\right)\right]\right\}}
$$


and

$$
A_{2}=-\left(v_{1} k_{l} / v_{2} k_{2}\right)\left[\sinh \left(k_{1} l / 2\right) / \sinh \left(k_{2} l / 2\right)\right] A_{1} .
$$

Now we write the temporal variation of the magnetic energy:

$$
\frac{\partial}{\partial t} U_{M}=2 i \omega_{0} h d\left[\int_{-l / 2}^{l / 2} u_{M}(z) d z\right] \exp \left(2 i \omega_{0} t\right) .
$$

We have $\mathscr{H}^{*}=H_{x}+i H_{y}$ and $H_{y}(z, t)=H_{y}(z) \exp \left(i \omega_{0} t\right)$. Then

$H_{x}^{2}(z, t)+H_{y}^{2}(z, t)=\frac{1}{2}\left(\mathscr{H}^{*}+\mathscr{H}^{*} \mathscr{H}\right)=\operatorname{Re}\left(\mathscr{H}_{\mathcal{H}^{*}}\right)=2 \overline{\mathcal{H}^{2}}$.

$\overline{H^{2}}$ is the temporal mean of $\mathscr{H}^{2}$ in a period $T=2 \pi / \omega_{0}$. To obtain the power dissipated as Joule heat, we use Ampère's law:

$$
\mathscr{E}=E_{x}-i E_{y}=-\frac{i c}{4 \pi \sigma_{c}} \frac{\partial \mathcal{H}}{\partial z} .
$$

We have taken $\sigma_{c}$ for the conductivity, since the microwave electric field is in the $c$-axis direction. In the configuration of Fig. 1 we have $E_{x}=0$ but we can also write in a general form the instantaneous power dissipat- ed as Joule heat:

$$
P_{J}=-\sigma_{c} h d\left[\int_{-l / 2}^{l / 2}\left[E_{y}^{2}(z)+E_{x}^{2}(z)\right] d z\right] \exp \left(2 i \omega_{0} t\right) .
$$

Defining $\mathscr{E}^{*}=E_{x}+i E_{y}$ we can write again

$$
\begin{aligned}
& E_{y}^{2}(z, t)+E_{x}^{2}(z, t)=\frac{1}{2}\left(\mathscr{E}^{*}+\mathscr{E}^{*} \mathscr{E}\right) \\
& =\operatorname{Re}\left(\mathscr{E}^{*}\right)=2 \overline{\mathscr{E}^{2}}
\end{aligned}
$$

$\overline{\mathscr{E}^{2}}$ is the temporal mean of $\mathscr{E}^{2}$ in a period $T$. Thus we write for the power absorbed, dropping the bar, and neglecting the electric energy:

$$
\begin{gathered}
\mathcal{P}=\operatorname{Re}\left[\frac{-i \omega_{0} h d}{2 \pi} \iint_{-l / 2}^{l / 2} 2 \mathcal{H}^{2} d z+4 \pi \int_{-l / 2}^{l / 2} 2 \mu \mathcal{H} d z\right] \\
\left.\times \frac{-\omega_{0} h d \delta^{2}}{4 \pi}\left[\int_{-l / 2}^{l / 2} 2 \mathscr{E}^{2} d z\right)\right]
\end{gathered}
$$

After carrying out the integration over $z$ and making the change of variables: $u=k_{1} l / 2, w=k_{2} l / 2$, and $\lambda=l / \delta$, we obtain

$$
\begin{gathered}
\mathcal{P}=\operatorname{Re}\left(( - \omega _ { 0 } h d l / 4 \pi ) \left\{i A_{1}^{2}\left(1+4 \pi v_{1}\right)[1+\sinh (2 u) /(2 u)]+A_{2}^{2}\left(1+4 \pi v_{2}\right)[1+\sinh (2 w) /(2 w)]\right.\right. \\
\left.+A_{1} A_{2}\left[2+4 \pi\left(v_{1}+v_{2}\right)\right][\sinh (u+w) /(u+w)+\sinh (u-w) /(u-w)]\right\} \\
+\left(1 / \lambda^{2}\right)\left\{2 A_{1}^{2} u^{2}[\sinh (2 u) /(2 u)-1]+2 A_{2}^{2} w^{2}[\sinh (2 w) /(2 w)-1]\right. \\
\left.\left.+2 A_{1} A_{2} u w[\sinh (u+w) /(u+w)-\sinh (u-w) /(u-w)]\right\}\right) .
\end{gathered}
$$

This expression can be simplified to give to first order in $\left(v_{1} / v_{2}\right)$ :

$$
\mathcal{P}=\operatorname{Re}\left(\frac{-i \omega_{0} h d l \mathcal{H}_{10}^{2}}{\pi \lambda^{2}}\left[\left(1-\frac{v_{1}}{v_{2}}\right)+\frac{v_{1}}{v_{2}} u^{2} \tanh ^{2}(u) \frac{\operatorname{coth}(w)}{w}\right]\right) .
$$

To first order in $\left(v_{1} / v_{2}\right)$ this expression is the same obtained from Kaplan's equation (14) taking tanh $(u) \rightarrow 1$ and $\operatorname{coth}(w) \rightarrow 1$ since $d \rightarrow \infty$ in Kaplan's asymmetrical case.

Now we can obtain the modulated power if we multiply expression (2) by a factor $A_{m} \cosh \left(k_{m} z\right)$, where $A_{m}$ is the amplitude of the modulation field and $k_{m}=(1+i) / \delta_{a}^{m}$. After carrying out the integration over $z$ we obtain the modulated power, taking $t=k_{m} l / 2=\frac{1}{2}(1+i) \lambda_{m}$, where $\lambda_{m}=l / \delta_{a}^{m}$ :

$$
\begin{aligned}
\mathcal{P}_{m}=\operatorname{Re}\left(\left(-i \omega_{0} h d l A_{m} / 4 \pi \lambda^{2}\right)\{\right. & 2 A_{1}^{2} u^{2}[\sinh (2 u+t) /(2 u+t)+\sinh (2 u-t) /(2 u-t)] \\
& +2 A_{2}^{2} w^{2}[\sinh (2 w+t) /(2 w+t)+\sinh (2 w-t) /(2 w-t)] \\
& +A_{1} A_{2}(u+w)^{2}[\sinh (u+w+t) /(u+w+t)+\sinh (u+w-t) /(u+w-t)] \\
& \left.\left.+A_{1} A_{2}(u-w)^{2}[\sinh (u-w+t) /(u-w+t)+\sinh (u-w-t) /(u-w-t)]\right\}\right) .
\end{aligned}
$$

\section{THE NORMAL LINE SHAPE}

To obtain the CESR line shape we have taken the derivative of expression (3) with respect to $\omega$ and we have plotted the line shapes in a computer as a function of $R$ and $\lambda$. As expected, we found the same line shapes of Dyson's theory or Kaplan's symmetrical case. Fitting the experimental spectra of GIC's in the configuration $\mathbf{H}_{0} \perp c$ (no magnetic-field-modulation-frequency effect) to the theoretical line shape allows us to determine the values of $R$ and $\lambda$. From the values of $\lambda$ and from $v_{0}$ (microwave frequency) and $l$ (width of sample) the $c$-axis resistivity can be obtained:

$$
\rho_{c}=\frac{l^{2} \pi v_{0} \mu_{0}}{\lambda^{2}}(\mathrm{mks}) \text {. }
$$

This procedure was applied by Walmsley et al. ${ }^{6}$ to $\mathrm{SbCl}_{5}$-intercalated GIC's and applied to $\mathrm{AlCl}_{3}$ intercalated GIC's by Walmsley and Rettori. ${ }^{7}$ 


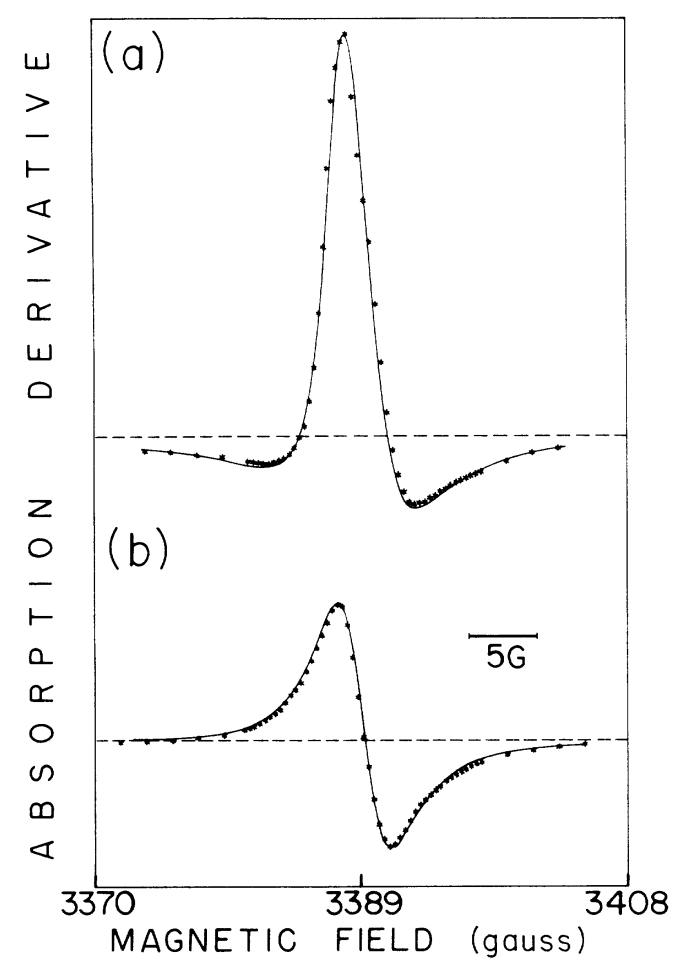

FIG. 2. (a) Room-temmperature CESR spectrum of stage-1 $\mathrm{AlCl}_{3}$-intercalated GIC for $\mathrm{H}_{0} \perp c(*)$ fitted to the normal line shape (solid line). Sample dimensions are $d=0.6 \mathrm{~mm}, l=2.2$ $\mathrm{mm}$, and $h=7.0 \mathrm{~mm}$. The fitted parameter is $\lambda=2.4$. (b) Room-temperature CESR spectrum of stage-1 $\mathrm{AlCl}_{3}$ intercalated GIC for $\mathbf{H}_{0} \| c$ and $v_{m}=100 \mathrm{kHz}(*)$ fitted to the modulation-effect line shape. The sample is the same as (a). The fitted parameter is $\lambda_{m}=4.4$ with $\lambda=2.4$.

\section{THE MODULATION-EFFECT LINE SHAPE}

The line shape obtained taking the derivative of expression (5) with respect to $\omega$ was computed as a function of $R, \lambda$, and $\lambda_{m}$. As expected, for small values of $\lambda_{m}$ $\left(\lambda_{m} \leq 0.1\right)$ the modulation-effect line shape coincides with the normal one. This result is in agreement with the fact that magnetic-field-modulation-frequency effects are not observed for low values of $v_{m}$. Also, the experimentally observed behavior of $A / B$ ratios, decreasing with increasing modulation frequency, ${ }^{2}$ appears from the calculated line shapes.

The CESR spectra of GIC's in the $\mathbf{H}_{0} \| c$ configuration which are affected by the magnetic-field-modulationfrequency effect, are fitted to the modulation-effect line shape, in order to determine the value of $\lambda_{m}$, since the values of $R$ and $\lambda$ must be determined from the spectra in the $\mathbf{H}_{0} \perp c$ configuration. From the values of $\lambda_{m}, v_{m}$ (magnetic-field-modulation-frequency) and $l$ (width of the sample) the in-plane resistivity can be obtained:

$$
\rho_{a}=\frac{l^{2} \pi v_{m} \mu_{0}}{\lambda_{m}^{2}}(\mathrm{mks})
$$

TABLE I. Room-temperature in-plane $\left(\rho_{a}\right)$ and $c$-axis $\left(\rho_{c}\right)$ resistivities in stage-1 $\mathrm{AlCl}_{3}$-intercalated GIC.

\begin{tabular}{cccc}
\hline \hline & $9.9 \pm 0.1$ & $3.8-4.6$ & $6.3-6.9$ \\
$\rho_{a}$ & CESR data & Ref. 9 & Ref. 8 \\
$(\mu \Omega \mathrm{cm})$ & $(100 \mathrm{kHz})$ & $(10-40 \mathrm{kHz})$ & $(\mathrm{dc})$ \\
& $3.1 \pm 0.1$ & $1.58-2.58$ & \\
$\rho_{c}$ & CESR data & Ref. 9 & \\
$(\Omega \mathrm{cm})$ & $(9 \mathrm{GHz})$ & $(\mathrm{dc})$ & \\
\hline \hline
\end{tabular}

\section{RESULTS FOR AICl ${ }_{3}$-INTERCALATED GIC'S}

The procedure described in this work for determining $\rho_{c}$ and $\rho_{a}$ was applied for the room-temperature spectra of a thick sample of stage- $1 \mathrm{AlCl}_{3}$-intercalated $\mathrm{GIC}$, in the $\mathbf{H}_{0} \perp c$ (no magnetic-field-modulation-frequency effect) and $\mathbf{H}_{0} \| c$ configurations, with modulation frequency of $100 \mathrm{kHz}$. For this sample we observed a very neat magnetic-field-modulation-frequency effect on the shape and on the intensity of the line. Moreover, the linewidth for this compound is homogeneous ${ }^{10}$ so we can rely on the information obtained from the line shape. Details of the sample preparation, characterization, and EPR measurements were described in Ref. 2.

Figure 2(a) shows the room-temperature spectrum of stage-1 $\mathrm{AlCl}_{3}$-intercalated GIC for $\mathbf{H}_{0} \perp c$ fitted to the normal line shape. The value of $\rho_{c}$ obtained from the $\lambda$ parameter is shown in Table $\mathrm{I}$. This value of $\lambda(\lambda=2.4)$ is in the range $(\lambda \leq 2.5)$ in which case the line shape does not depend on the $R$ parameter. ${ }^{3}$

Figure 2(b) shows the room-temperature spectrum of the same sample of stage- $1 \mathrm{AlCl}_{3}$-intercalated $\mathrm{GIC}$ for $\mathbf{H}_{0} \| c$ and $v_{m}=100 \mathrm{kHz}$ fitted to the modulation-effect line shape. From the parameter $\lambda_{m}$ we calculate $\rho_{a}$ (Table I). The value of $\lambda$ was taken from the fitting of Fig. 2(a).

\section{CONCLUSIONS}

We have shown that the magnetic-field-modulationfrequency effect on the CESR line shape of graphite intercalation compounds in the $\mathbf{H}_{0} \| c$ configuration can be obtained theoretically, using Kaplan's theory as a starting point. We show that the in-plane resistivity can be obtained from a fitting of the experimental spectrum with the theoretical line shape. The values of in-plane and $c$ axis resistivity that we obtained for our sample of stage-1 $\mathrm{AlCl}_{3}$-intercalated GIC from CESR measurements are in reasonable agreement with the values obtained by Bach and McRae for their samples from dc and inductive methods.

\section{ACKNOWLEDGMENT}

This work was partially supported by Fundação de Amparo a Pesquisa do Estado de São Paulo, Brazil. 
${ }^{1}$ R. M. Stein, L. Walmsley, and C. Rettori, Phys. Rev. B 32, 4134 (1985).

${ }^{2}$ L. Walmsley, G. Ceotto, J. H. Castilho, and C. Rettori, Synth. Met. 30, 97 (1989)

${ }^{3}$ F. J. Dyson, Phys. Rev. 98, 349 (1955).

${ }^{4}$ M. Saint Jean, C. Rigaux, J. Blinowski, B. Clergaud, P. Kacman, and G. Furdin, Ann. Phys. (Paris) 11, 215 (1986).

${ }^{5}$ J. I. Kaplan, Phys. Rev. 115, 575 (1959).

${ }^{6}$ L. Walmsley, S. Rolla, and C. Rettori, Synth. Met. 23, 305
(1988).

${ }^{7}$ L. Walmsley and C. Rettori, Synth. Met. 36, 235 (1990)

${ }^{8}$ B. Bach and A. R. Ubbelohde, Proc. R. Soc. London Ser. A 325, 437 (1971).

${ }^{9}$ E. McRae, J. F. Marêché, P. Pernot, and R. Vangelisti, Phys. Rev. B 39, 9922 (1989).

${ }^{10}$ A. M. Gennaro, J. C. Sartori, C. Rettori, and G. E. Barberis, Materials Science Forum (Trans Tech, New York, 1992), Vols. 91-93, p. 533. 